\title{
Optimal Design of Feedback Control by Inhibition
}

\author{
Steady State Considerations
}

\section{Michael A. Savageau}

Department of Microbiology, The University of Michigan, Ann Arbor, Michigan 48104

Received April 9, 1974

Summary. A recent1y developed, nonlinear method of systems analysis has been used to compare alternative patterns of control by feedback inhibition in otherwise equivalent unbranched biosynthetic pathways. The steady state performance of the simple case with end-product inhibition at the first physiologically important step is optimal with respect to the following criteria: the ability to (i) meet an increased demand for the end-product, (ii) meet this increased demand with limited accumulation of the intermediates, (iii) respond to an increased supply of the initial substrate, and (iv) limit the accumulation of the intermediates while responding to the increased availability of the initial substrate. The importance of these properties for the selection of feedback patterns in biosynthetic pathways is discussed.

Key words: Natural Selection - Control Patterns - Biosynthetic Pathways.

\section{Introduction}

The existence of control by feedback inhibition in the biosynthetic pathways of microorganisms has been known for more than 15 years (Umbarger, 1956, Yates \& Pardee, 1956). Since these initial discoveries, numerous examples of this type of control have been reported. On the basis of the empirical results Monod, Changeux and Jacob (1963) have postulated three rules that appear to govern the pattern of feedback interactions in unbranched biosynthetic pathways: ( $i)$ the end-product in such a pathway acts as an allosteric inhibitor of the first reaction in the sequence, (ii) the intermediate metabolites do not modify the first reaction, and (iii) the end-product does not modify the activity of the intermediate enzymatic reactions.

At present there is no satisfactory explanation for this pattern of control being the most prevalent among a11 the possible patterns. In fact, to date, 
the natural selection of control patterns in unbranched biosynthetic pathways has not been systematica11y examined experimenta11y or theoretically. Koch (1967) has used classical steady state enzyme kinetics to examine the possible advantages of direct inhibition of an enzyme by its product for the regulation of metabolic pathways. Although this classical description of single enzymes may be an accurate approximation in many cases, the mathematics are cumbersome, and the treatment of large numbers of such reactions or the comparison of alternative patterns quickly becomes unwieldy.

While in principle the behavior of a complex biochemical system can be related to the nature of its component enzymatic reactions, in practice the analysis of such systems is made extremely difficult because the rate laws that characterize the individual reactions are rational functions (Wong \& Hanes, 1962). One approach to circumvent the mathematical difficulties in analyzing such systems is linearization. It is well known that a linear approximation to the nonlinear rate law is a valid and accurate representation of the original rate law as long as the concentration variables do not deviate appreciably from the operating values selected for the approximation. The disadvantage of this approach is that the linear approximation is only valid over a restricted range in the concentration variables, and this range is not sufficiently large to be useful for most biochemical systems that are highly nonlinear.

The general philosophy of approximation described above can be extended to curvilinear approximations that remain valid over a much wider range in concentration values. To be useful such nonlinear approximations must be based on the essential nonlinearity of the rate law and yet be sufficiently simple to treat mathematically. The first requirement is to ensure the validity over a wide range of concentration values; the second is necessary if we are to deal with large numbers of reactions interrelated in complex ways.

I have described a power-law approximation that appears to meet these requirements (Savageau, 1969). By means of a logarithmic transformation and a Taylor series expansion one can prove that a power-law approximation to the nonlinear rate law is also a valid and accurate representation of the original rate law when the excursions of the concentration variables from the normal operating values are small. However, "sma11" in this instance is considerably greater than it is in the case with linearization; in fact, it appears large enough to be physiologically relevant (Savageau, 1969, 1971). A direct experimental validation of the power-law approximation is provided by the work 
of Kohen \& Kohen (1972) in which they have examined the kinetics of individual reactions in vivo. They use a microelectrode to inject by electrophoresis substrate of known concentration into a localized region of an individual cell. The resulting rate is obtained by microfluorimetrically recording the changes in the oxidation-reduction state of the cofactor to which the substrate conversion is coupled. With these techniques they have demonstrated power-law kinetics for the enzymes of the glycolytic sequence.

The specific concepts, terminology and symbols associated with this formalism are perhaps most easily understood in the context of specific examples. Several different types of examples, as well as a more detailed description of the power-1aw approach, its justification and merits, are given elsewhere (Savageau, 1972). The relevant concepts, terminology and symbols of this formalism also will be described during the course of the analysis that fo11ows.

In this paper I shall examine unbranched biosynthetic pathways subject to control by various patterns of feedback inhibition. These systems can be shown to have a unique steady-state solution in general (Savageau, 1969). The conditions for the local stability of this solution will be examined in a subsequent paper;for the present we shall simply assume these conditions are satisfied and emphasize the steady-state properties of these systems. The results of this analysis provide the first explanation for the experimentally observed predominance of the simple end-product inhibition pattern in nature.

Pathways Controlled by End-Product Inhibition

Since this pattern of control is of paramount importance in the natural state, we should thoroughly understand its behavior. This specific example will also best illustrate the essentials of the analysis which follows, and the generalization to other patterns of control can then be treated with a minimum of detail. Consider the pathway represented in Fig. la. We will assume that the enzyme levels remain constant over the time scale of interest in this paper and thus deal only with metabolic regulation.

According to the previously described analytical technique, each rate law is approximated by a product of power functions, one for each of the reactants and modifiers associated with the reaction. For the system represented in Fig. 1a the following equations apply. 


$$
\frac{d X_{1}}{d t}=\alpha_{1} x_{0}{ }^{g_{10}} x_{n}{ }^{g} 1 n-\beta_{1} x_{1}{ }^{h} 11
$$

(1)

$$
\begin{aligned}
& \frac{\mathrm{dx}_{2}}{\mathrm{dt}}=\alpha_{2} \mathrm{x}_{1}{ }^{\mathrm{g}_{21}}-\beta_{2} \mathrm{x}_{2}{ }^{\mathrm{h}_{22}} \\
& \vdots \\
& \frac{\mathrm{dx}}{\mathrm{dt}}=\alpha_{n} \mathrm{x}_{\mathrm{n}-1} \mathrm{~g}_{\mathrm{n}, \mathrm{n}-1}-\beta_{\mathrm{n}} \mathrm{x}_{\mathrm{n}}^{\mathrm{h}_{\mathrm{n}}}
\end{aligned}
$$

The exponent $g_{i, i-1}$ represents the apparent kinetic order with respect to $x_{i-1}$, for the synthesis of $x_{i}$, and $\alpha_{1}$ is the apparent rate constant for this reaction. The description of the degradative terms is similar except the corresponding parameters are $h_{i i}$ and $B_{i}$, respectively, and the substrate for these reactions is $x_{i}$ rather than $x_{i-1} \cdot g_{i n}$, the apparent kinetic order of the first reaction with respect to $x_{n}$, is negative since $\mathrm{X}_{\mathrm{n}}$ is an inhibitor; all other parameters of this system are positive.

\section{Steady_State Solutions}

The steady state solutions for Eqs. (1), when the time derivatives are equal to zero, are intrinsically important for describing the behavior of many biochemical systems that normally operate in the steady or quasisteady state. In the present case, the steady state equations can be simplified because the rate of utilization of $x_{i}$ is the same as the rate of production of $x_{i+1}$, and the resulting nonlinear algebraic equations can be transformed into a set of linear equations to give the following results:

$$
\mathrm{b}_{1}=\mathrm{g}_{10} \mathrm{y}_{0}-\mathrm{h}_{11} \mathrm{y}_{1}+\mathrm{g}_{1 \mathrm{n}} \mathrm{y}_{\mathrm{n}}
$$

$$
\begin{aligned}
& \mathrm{b}_{2}=\mathrm{h}_{11} \mathrm{y}_{1}-\mathrm{h}_{22} \mathrm{y}_{2} \\
& \vdots \\
& \mathrm{b}_{\mathrm{n}}=\mathrm{h}_{\mathrm{n}-1, \mathrm{n}-1} \mathrm{y}_{\mathrm{n}-1}-\mathrm{h}_{\mathrm{nn}} \mathrm{y}_{\mathrm{n}}
\end{aligned}
$$

where

$$
\begin{aligned}
& b_{1}=\log \left(\beta_{1} / \alpha_{1}\right) \\
& b_{i}=\log \left(\beta_{i} / \beta_{i-1}\right) \quad \text { for } i>1 \\
& y_{i}=\log \left(x_{i}\right)
\end{aligned}
$$

These linear equations can be solved for the dependent variables in terms of the independent variable $y_{0}$ and the various parameter values, providing 
the determinant is non-zero. The resulting solutions are

$$
y_{i}=\frac{\left(g_{10} y_{0}-b_{1}-b_{2}-\ldots-b_{i}\right) h_{n n}-\left(b_{i+1}+b_{i+2}+\ldots+b_{n}\right) g_{1 n}}{h_{i i}\left(h_{n n}-g_{1 n}\right)}
$$

where $b_{j} \equiv 0$ for all $j>n$.

These results can be easily transformed back to give the steady state concentrations of the intermediates as functions of the independent concentration $\mathrm{x}_{0}$ and the system's parameter values. Eqs.(3) also form the basis upon which further analysis is developed.

Responses to Change in the_Initial Substrate_or End-Product

From Eqs.(3) we can obtain the percentage change in the dependent concentrations in response to a percentage change in $x_{0}$ by simply taking the partial derivatives with respect to $y_{0}$. These partial derivatives have been previously defined as logarithmic-gain factors (Savageau, 1971). Thus,

$$
\begin{array}{r}
\mathrm{L}_{i 0}=\frac{\partial \mathrm{y}_{i}}{\partial \mathrm{y}_{0}}=\frac{\mathrm{h}_{\mathrm{nn}}}{\left(\mathrm{h}_{\mathrm{nn}}{ }^{\left.-g_{1 n}\right)} \frac{g_{10}}{h_{i i}}\right.} \\
i=1,2, \ldots, \mathrm{n}
\end{array}
$$

These partial derivatives are all positive since all the parameters are positive except $g_{1 n}$ which is negative. In other words, all the dependent concentration variables will increase in response to an increase in $\mathrm{x}_{0}$.

The changes in the dependent concentrations in response to an alteration in the exogenous supply of the end product can be investigated in an analogous fashion. This exogenous pool of $x_{n}$ will be denoted by $x_{n}$. To account for the contribution of $x_{n}$, to the rate of increase in the endogenous equivalent $x_{n}$, we must include an additional power function of the form $x_{n}{ }^{\prime} n^{\prime}$ in the synthetic term of the last of Eqs.(1). The steady state solutions analogous to Eqs.(2) can then be obtained in exactly the same manner, and from these solutions we calculate the following logarithmic-gains: 
(5)

$$
\begin{aligned}
& L_{i n^{\prime}}=\frac{\partial y_{i}}{\partial y_{n^{\prime}}}= \frac{g_{n n^{\prime}} g_{1 n}}{h_{i i}\left(h_{n n}-g_{1 n}\right)} \\
& i=1,2, \ldots, n^{-1} \\
&=\frac{g_{n n^{\prime}}}{\left(h_{n n^{-g}} g_{1 n^{\prime}}\right.} \\
& i=n
\end{aligned}
$$

These expressions give the percentage change in the intermediate concentrations in response to a one percent change in the exogenous supply of the end-product. They are a11 negative quantities (except $\mathrm{L}_{\mathrm{nn}}{ }^{\prime}$ ) indicating a decrease in response to an increase in $x_{n}$,. Thus, the larger the magnitude of the logarithmic-gain $\mathrm{L}_{i n}$, the greater the sparing effect on the $i^{\text {th }}$ intermediate, and for all $i$ this is achieved by increasing the strength of inhibition $\mathrm{g}_{1 \mathrm{n}}$.

Responses to a Change in Demand for the End-Product or Parameter Variations

The logarithmic-gain factors in Eqs. (4) and (5) are properties of the intact system. The sensitivity of these system properties to variations in parameters characterizing the component parts of the system is defined as

$$
S_{L p}=\frac{\partial L}{\partial p} \frac{p}{L}
$$

where $L$ can be any logarithmic-gain factor of the system and $P$ any component parameter. For example, the sensitivity of the overall gain, $\mathrm{L}_{\text {no }}$, with respect to a change in $h_{n n}$, the apparent kinetic order of the final reaction with respect to the concentration of the end-product, is given by

$$
\mathrm{S}_{\mathrm{L}_{\mathrm{nO}} \mathrm{h}_{\mathrm{nn}}}=\frac{\partial \mathrm{L}_{\mathrm{nO}}}{\partial \mathrm{h}_{\mathrm{nn}}} \frac{\mathrm{h}_{\mathrm{nn}}}{\mathrm{L}_{\mathrm{nO}}}=-\mathrm{h}_{\mathrm{nn}} /\left(\mathrm{h}_{\mathrm{nn}}-\mathrm{g}_{1 \mathrm{n}}\right)
$$

This is the most important of the parameter sensitivities because $h_{n n}$ represents the "elasticity of demand" for the end-product $x_{n}$. Any of a wide variety of modifications in cellular metabolism could change the demand function for $x_{n}$ (i.e. the rate law for the utilization of $x_{n}$ ), and therefore would be reflected in a change of $h_{\mathrm{nn}}$. Similarly, the sensitivities of the intermediate logarithmic-gains with respect to a change in $h_{n n}$ are 
(8)

$$
\mathrm{S}_{\mathrm{L}_{\mathrm{i} 0} \mathrm{~h}_{\mathrm{nn}}}=-\mathrm{g}_{1 \mathrm{n}} /\left(\mathrm{h}_{\mathrm{nn}}-\mathrm{g}_{1 \mathrm{n}}\right) \quad \mathrm{i}=1,2, \ldots, \mathrm{n}-1
$$

The sensitivity in Eq.(7) is always negative while those in Eq.(8) are always positive. This is in agreement with our intuition; an increase in the demand for the end-product would deplete the end-product concentration and, by the release of inhibition, increase the intermediates.

Other sensitivities could also be examined in this way. However, the above are the most important for characterizing the performance of the control system in the cell, and it is these we shall make use of in the following sections.

Optimal Pattern of Control with Respect to Variations in the End-Product

Other patterns of feedback interaction could also be considered that in principle possess the ability to regulate the supply of the end-product. Four of the many possibilities are represented in Fig. 1, cases (b) - (e). There have been few criteria for comparing and evaluating such regulatory alternatives other than a description of the molecular mechanisms and the topology of the feedback interactions. Consequently, it has been difficult to distinguish these systems on the basis of function, and thus to explain the natural prevalence of the pattern in Fig.la. The quantitative techniques illustrated in the preceding section provide a novel approach to this question.

(a)

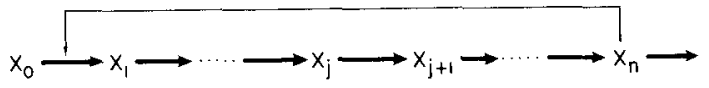

(b)

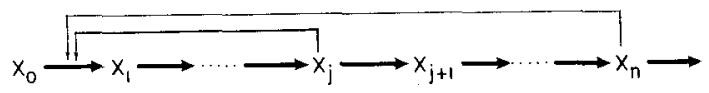

(c)

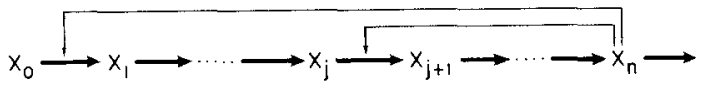

(d)

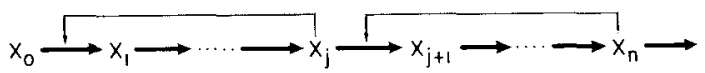

(e)

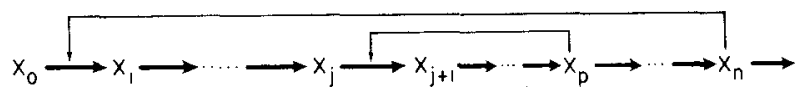

Fig. l a - e. Alternative patterns of feedback control by inhibition in unbranched biosynthetic pathways 
A general unbranched sequence with $n+1$ metabolites and with an arbitrary pattern of feedback inhibitions is governed by the following steady state equation in logarithmic form:

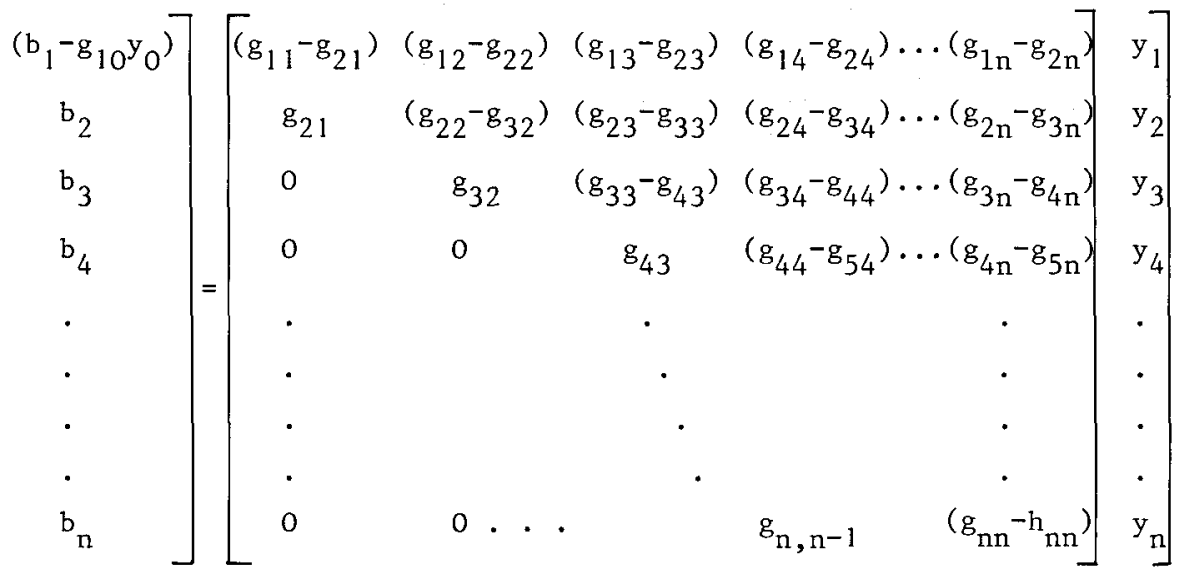

This equation is analogous to Eq.(2), but it applies to reversible as well as irreversible reactions. The logarithmic-gain $\mathrm{L}_{\mathrm{nO}}$ for this system is given by

$$
L_{n 0}=\frac{\partial y_{n}}{\partial y_{0}}=\frac{(-1)^{n} g_{n, n-1} \ldots g_{43} g_{32} g_{21} g_{10}}{\Delta_{n}}
$$

where $\Delta_{n}$ is the determinant of the matrix in Eq. (9). The logarithmic-gain for the system in Fig.la will be written as

$$
\mathrm{L}_{\mathrm{nO}}^{\mathrm{a}}=\mathrm{g}_{10} /\left(\mathrm{h}_{\mathrm{nn}}-\mathrm{g}_{1 \mathrm{n}}\right)
$$

where the superscript "a" refers to the specific system under consideration.

To restrict the comparison of these two systems to the differences in their pattern of control we must require that the sequences be equivalent in all other respects. The only differences between the systems that will be allowed are those involving the parameter values directly related to the differences in their pattern of control. In particular, all common parameters must have identical values and the logarithmic-gains of the overall systems must be identical, i.e., $\mathrm{L}_{\mathrm{nO}}^{\mathrm{a}}=\mathrm{L}_{\mathrm{nO}}$. From Eqs. (10) and (11)

$$
1 /\left(h_{n n}-g_{1 n}^{a}\right)=(-1)^{n} g_{n, n-1} \ldots g_{43} g_{32} g_{21} / \Delta_{n}
$$

where the superscript "a" identifies the distinctive parameter for the system in Fig.la. Under this constraint a given change in the logarithm of the input 
concentration of each sequence results in the same logarithmic increment for the output concentrations of the two systems.

Having established the necessary relationships between the systems to be compared, one can determine the sensitivities with respect to variations in their common parameters. The sensitivity of $\mathrm{L}_{\mathrm{nO}}$ with respect to changes in the parameter $h_{n n}$ is given by

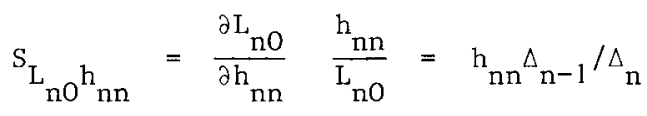

where $\Delta_{n-1}$ is the determinant remaining when the last row and column of $\Delta_{n}$ have been eliminated. It is also the determinant of the matrix characteristic of the system with $n$ metabolites. The sensitivity of $\mathrm{L}_{\text {no }}^{\mathrm{a}}$ with respect to changes in the parameter $h_{n n}$ is

$$
\mathrm{S}_{\mathrm{L}_{\mathrm{nO}}^{\mathrm{a}} \mathrm{h}_{\mathrm{nn}}}=-\mathrm{h}_{\mathrm{nn}} /\left(\mathrm{h}_{\mathrm{nn}}-\mathrm{g}_{1 \mathrm{n}}^{\mathrm{a}}\right)
$$

and by utilizing the identity in Eq. (12) this equation becomes

$$
\mathrm{S}_{\mathrm{L}_{\mathrm{nO}}^{\mathrm{a}} \mathrm{h}_{\mathrm{nn}}}=-\mathrm{h}_{\mathrm{nn}}(-1)^{\mathrm{n}} \mathrm{g}_{\mathrm{n}, \mathrm{n}-1} \ldots \mathrm{g}_{43} \mathrm{~g}_{32} \mathrm{~g}_{21} / \Delta_{\mathrm{n}}
$$

To compare these sensitivities we take the ratio of the two expressions in Eqs. (13) and (15).

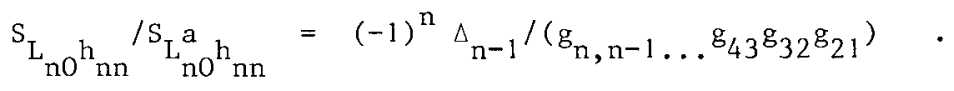

Finally, by expanding the determinant $(-1)^{n-1} \Delta_{n-1}$ we find

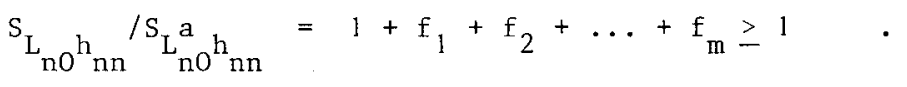

The ratio of sensitivities in Eq. (17) is always greater than or equal to one because (i) each of the terms in the expansion of the numerator in Eq. (16) is non-negative, (ii) one of these terms is equal to the denominator, and (iii) the denominator is positive (see "Appendix"). Thus, each of the fractions $f_{i}$ is non-negative. The ratio having a value greater than or equal to unity indicates that no pattern of feedback inhibition can render the system less sensitive to this parameter variation than the simple pattern in Fig.la. 
Although minimization of a parameter sensitivity has been emphasized in this section, this is only one of the many advantages obtained with the simple pattern of control by end-product inhibition. For example, if the sensitivities of the different systems are made equal for purposes of comparison, then the overall gain $\mathrm{L}_{\text {no }}$ is maximized by the pattern of control in Fig.la. This can easily be seen from an examination of Eqs.(10) and (13), and (11) and (14). Since the ratio of $L_{\text {no }}^{a}$ to $S_{L_{n}^{a} h_{n n}}$ is a constant, raising the value of the previously minimal sensitivity, $\mathrm{S}_{\mathrm{L}_{n O} \mathrm{~h}_{\mathrm{n}}}$, to equality with $\mathrm{S}_{\mathrm{L}_{\mathrm{n}} \mathrm{h}_{\mathrm{nn}}}$, simultaneously makes $L_{n 0}^{a}$ greater than or equal to $L_{n 0}$. The optimization of $\mathrm{S}_{\mathrm{L}_{\mathrm{n}} \mathrm{h}_{\mathrm{nn}}}$, which reflects the ability of the system to meet an increased demand for the end-product, and $\mathrm{L}_{\text {no }}$, which measures the ability of the system to translate an increased availability of initial substrate into endproduct, has important physiological implications that will be treated further in the "Discussion" section.

\section{Optima1 Pattern of Control with Respect to Variations in the Intermediates}

The levels of the intermediate metabolites in an unbranched pathway will generally increase in response to an increase in the supply of the initial substrate. For example, this is evident from Eqs. (4) for a system employing simple end-product inhibition. As Atkinson (1969) has pointed out, these increases in the levels of metabolic intermediates must be minimized because there is a limited solvent capacity in the cell. For responsive control in pathways producing substances needed in only small quantities it is also necessary to limit the accumulation of the intermediates (Koch, 1967). It is therefore of interest to compare the magnitudes of these increases in systems employing various patterns of control by inhibition.

The logarithmic-gain $\mathrm{L}_{i 0}$ represents the change in the logarithm of the $i^{\text {th }}$ intermediate in response to a unit change in the logarithm of the initial substrate $x_{0}$. This gain can be calculated from Eq.(9) for a system with an arbitrary pattern of control. The result of this calculation is

$$
L_{i 0}=(-1)^{i} g_{10}\left[\prod_{j=1}^{i-1} g_{j+1, j}\right] \frac{\Delta_{n-i}}{\Delta_{n}} \quad i=1,2, \ldots n-1
$$

where $\Delta_{n}$ is the determinant of the entire matrix in Eq. (9), $\Delta_{n-i}$ is the 
subdeterminant remaining when the first $i$ rows and columns of $\Delta_{n}$ are deleted, and

$$
\prod_{j=1}^{0} g_{j+1, j} \equiv 1
$$

Similarly, for the system in Fig.la

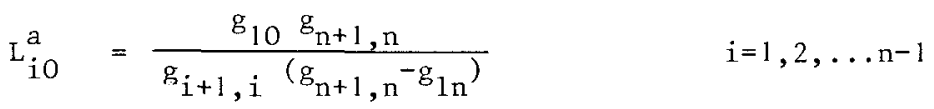

where $g_{n+1, n}$ is by definition the same as $h_{n n}$.

In comparing the case of Fig.la with an arbitrary pattern of control we shall require that the two systems be equivalent in all respects except for those directly related to the differences in the patterns of control. In particular, all common parameters must be equal and the overall gains must be the same, i.e., $\mathrm{L}_{\mathrm{nO}}^{\mathrm{a}}=\mathrm{L}_{\mathrm{nO}}$. These are the same conditions for equivalence that were used in the previous section. Accordingly, these conditions lead to the constraint expressed in Eq.(12). Using this constraint allows us to rewrite $\mathrm{Eq} \cdot(19)$ as

$$
\mathrm{L}_{\mathrm{i} 0}^{\mathrm{a}}=\frac{(-1)^{\mathrm{n}} \mathrm{g}_{10} \prod_{j=1}^{\mathrm{n}} \mathrm{g}_{j+1, j}}{\mathrm{~g}_{\mathrm{i}+1, i} \Delta_{n}}
$$$$
\mathrm{i}=1,2, \ldots \mathrm{n}-1 \quad \text {. }
$$

We can now compare the logarithmic-gains for these two systems by taking the ratio of the expressions in Eqs.(18) and (20).

$$
\mathrm{L}_{i 0} / \mathrm{L}_{\mathrm{i} O}^{\mathrm{a}}=(-1)^{\mathrm{n}-\mathrm{i}} \Delta_{\mathrm{n}-\mathrm{i}} / \prod_{j=i+1}^{\mathrm{n}} \mathrm{g}_{j+1, j} \geq 1
$$

$$
i=1,2, \ldots n-1 \quad \text {. }
$$

This ratio has exactly the same properties as the ratio in Eq.(16), as can be demonstrated by simply renumbering the indices. The results in Eq. (21) may be sumnarized as follows. For a given increase in the initial substrate concentration $x_{0}$, the resulting increase in the level of the end-product $x_{n}$ will be identical for the system in Fig.la and an otherwise equivalent system with an arbitrary pattern of contro1. However, the system in Fig.1a can achieve this increase in $x_{n}$ with a minimum elevation in the concentrations of its intermediate metabolites. 
The sensitivities of these intermediate logarithmic-gains, with respect to a change in $g_{n+1, n}$ (or $h_{n n}$ ), can also be calculated. The resulting expression provides a measure for the effect of end-product demand on the change in levels of the intermediates. For a system with an arbitrary pattern of control, the sensitivity of the $i^{\text {th }}$ logarithmic-gain with respect to changes in $g_{n+1, n}$ is calculated from Eq. (18).

$$
S_{L_{i 0}} g_{n+1, n}=\frac{g_{n+1, n} \Delta_{n-1}}{\Delta_{n}}-\frac{g_{n+1, n} \Delta_{n-i-1}}{\Delta_{n-i}} \quad i=1,2, \ldots n-1
$$

where $\Delta_{n-1}$ is the subdeterminant remaining when the last row and column of $\Delta_{n}$ are deleted, and $\Delta_{n-i-1}$ is the subdeterminant remaining when the last row and column of $\Delta_{n-i}$ are deleted. The first term on the right hand side of this equation is equal to $S_{L_{n O}} g_{n+1, n}$, as can be seen from Eq. (13). Therefore

$$
s_{L_{i 0}} g_{n+1, n}=S_{L_{n 0}} g_{n+1, n}-\frac{g_{n+1, n} \Delta_{n-i-1}}{\Delta_{n-i}} \quad i=1,2, \ldots n-1 \text {. }
$$

Similarly, for the system in Fig.la, the corresponding sensitivities can be calculated from Eq. (19). Thus,

$$
S_{L_{i 0}^{a}} g_{n+1, n}=\frac{-g_{n+1, n}}{g_{n+1, n}-g_{1 n}^{a}}+1 \quad i=1,2, \ldots n-1
$$

or

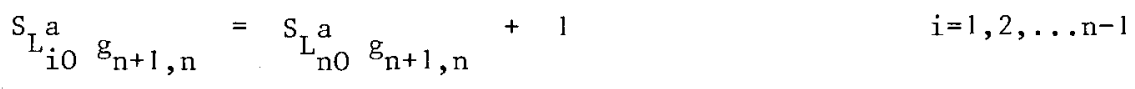

where we have made use of Eq. (14).

Again we can compare these sensitivities by taking the ratio of the expressions in Eqs. (23) and (25):

$$
\frac{S_{L_{i 0}} g_{n+1, n}}{S_{L_{i 0}^{a}}^{a} g_{n+1, n}}=\frac{s_{L_{n O}} g_{n+1, n}}{S_{L_{n O}^{a}}^{a} g_{n+1, n}+\frac{g_{n+1, n} \Delta_{n-i-1}}{\Delta_{n-i}}} \frac{1}{1} \quad i=1,2, \ldots n-1 \text {. }
$$

In this equation

$$
S_{L_{n O}} g_{n+1, n} \geq S_{L_{n O}^{a}}^{a} g_{n+1, n},
$$


but at the same time $-g_{n+1, n} \Delta_{n-i-1} \leq \Delta_{n-i}$, so no general conclusion regarding the magnitude of the ratio in $\mathrm{Eq}$. (26) can be reached. The results will depend upon the particular parameter values for the systems involved in the comparison. In this sense, there is no optimal pattern of control that is independent of the specific parameter values. Nevertheless, there is a subclass of systems for which the case in Fig.la is least sensitive. When the end-product inhibits no reaction after the $j^{\text {th }}$, then

$$
-g_{n+1, n} \Delta_{n-i-1}=\Delta_{n-i}
$$

and Eq. (26) becomes

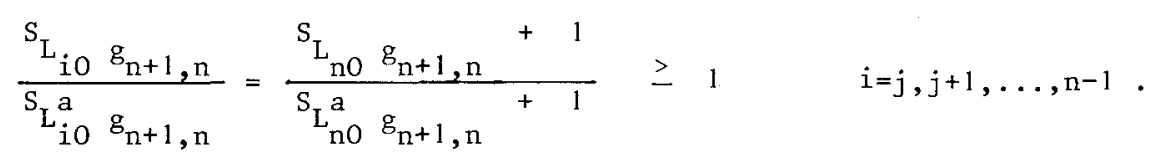

Under these conditions the ratio in Eq.(27) is greater than or equal to one, since

$$
\mathrm{S}_{\mathrm{L}_{\mathrm{n} 0}} \mathrm{~g}_{\mathrm{n}+1, \mathrm{n}} \geq \mathrm{S}_{\mathrm{L}_{\mathrm{n} 0}^{\mathrm{a}}} \mathrm{g}_{\mathrm{n}+1, \mathrm{n}}
$$

For the class of systems in which the end-product only inhibits the first reaction, the case represented in Fig.la is optimal because the sensitivities for all the intermediates in the pathway are minimally sensitive.

The following considerations indicate that this criterion may be less important than the others we have considered. From Eqs. (19) and (24) we see that $L_{i 0}^{a}$ is a decreasing function of $g_{1 n}^{a}$, while $S_{L_{i 0}^{a}} g_{n+1, n}$ increases and reaches a maximum value of 1 as $g_{1 \mathrm{n}}^{a}$ is increased. Thus, when $\mathrm{S}_{\mathrm{L}_{\text {io }}}^{\mathrm{a}} \mathrm{g}_{\mathrm{n}+1, \mathrm{n}}$ is large, implying a large percentage variation in $\mathrm{L}_{\text {io }}^{\mathrm{a}}$, $\mathrm{L}_{\text {io }}^{\mathrm{a}}$ is small; the net variation in $\mathrm{L}_{i 0}^{a}$ is still small for the system in Fig.la. In fact, one can show that the absolute variations in the $\mathrm{L}_{i 0}$ are minimal for the system in Fig.la. This is done by multiplying the expressions in Eqs. (21) and (26) and showing that the result is always greater than or equal to one. Thus, the product of $\mathrm{L}_{i 0}^{\mathrm{a}}$ and $\mathrm{S}_{L_{i 0}}^{a} \mathrm{~g}_{\mathrm{n}+1, n}$ is minimal. The smal1 value of $L_{i 0}^{a}$ compensates for the large magnitude of $S_{L_{i 0}} g_{n+1, n}$ and the system functions we11, even under conditions where these sensitivities are not themselves minima1. 
One of the primary functions of a control system for a biosynthetic pathway is to maintain a relatively constant supply of the end-product for macromolecular synthesis. Such a system must continue functioning in spite of both internal and external changes if it is to remain viable.

Internal variations are those affecting the kinetic parameters of the components within the system itself. These changes may be the direct result of mutation, errors in transcription or translation of the genetic information, or environmental influences such as temperature shifts, etc. The ability of the system to perform under these conditions would indicate that it is relatively "insensitive" to these perturbations. Indeed, as we have already seen, "minimum sensitivity" appears to be an important criterion for the natural selection of feedback patterns in biosynthetic pathways. Although these sources of internal variation are important to consider, most of the variations would be expected to occur outside of the system proper, and these would have their effect upon the system indirectly. These indirect effects are of two types: those affecting the demand for the end-product and those affecting the supply of the initial substrate.

A wide variety of external conditions will lead to a capacity for an increased rate of protein synthesis. Indirectly, these changes alter the demand function for the end-product, and this is reflected in a change of $h_{n n}$, as mentioned in the preceding sections. Thus, minimizing the sensitivity of the end-product (or the overall gain $\mathrm{L}_{\mathrm{n}}$ when the initial substrate is assumed to be constant) with respect to variations in the apparent kinetic order of the end-product, $h_{n n}$, has special significance here. Those systems that are minimally sensitive can best maintain the level of the end-product in the face of this demand and will allow protein synthesis to occur at the greatest rate. The organisms utilizing these systems will be best able to take advantage of the prevailing conditions and grow at the fastest rate.

On the other hand, there are several conditions external to the system that can be expected to increase the supply of the initial substrate $x_{0}$. The systems most efficient in utilizing the additional substrate would produce more end-product and thereby exert a correspondingly greater influence to increase the rate of protein synthesis. In other words, organisms that have a system with a greater overall gain, $\mathrm{L}_{\mathrm{no}}$, would tend to have a selective advantage under this set of conditions. 
It is clear that the low sensitivity desired for the first class of external perturbations and the high gain desired for the second class are actually in conflict. This can be seen from the last of Eqs. (4) and (7). If $\mathrm{S}_{L_{n O}} h_{n n}$ is decreased, by increasing the strength of inhibition $g_{1 n}$, $L_{n o}$ must correspondingly decrease. Conversely, increasing $\mathrm{L}_{\mathrm{no}}$ will simultaneously increase $\mathrm{S}_{\mathrm{L}_{\mathrm{nO}}} \mathrm{h}_{\mathrm{nn}}$. Thus the ratio $\mathrm{L} / \mathrm{S}$ is relatively constant, and it is this "figure of merit" for a biosynthetic system that is maximized by the end-product pattern of inhibition. This means that a compromise between logarithmic-gain and desensitization must be made; but whatever balance is established, the simple end-product inhibition pattern assures the maximum of both advantages.

Although the simple pattern of end-product inhibition maximizes $\mathrm{L} / \mathrm{S}$, there are other patterns that also possess this property, e.g., cases (c) and (e) in Fig.1. However, these do not appear to be found among unbranched biosynthetic pathways in nature. The joint probability of two or more regulatory enzymes evolving by random mechanisms is considerably less than for a single regulatory enzyme. Thus if they did exist, cases such as (c) and (e) initially might have constituted an infinitesimal percentage, in comparison to case (a), of the population in which they evolved. Other things being equal, cases such as these would remain a minute percentage of the population in comparison to case (a), or be easily lost by mutation to case (a), since they have no selective advantage based on the criterion of minimum sensitivity. Furthermore, these other patterns in general are not optimal with respect to the other two criteria we have considered.

Accumulation of the metabolic intermediates in a biosynthetic pathway can commonly result from external changes which increase the supply of the initial substrate or from those that increase the demand for the end-product. When equivalent systems utilizing alternative patterns of inhibition are compared on the basis of these two criteria, the $\mathrm{L}_{\mathrm{i} 0}$, and the ${ }^{\mathrm{S}_{\mathrm{L}}}{ }_{\mathrm{iO}} \mathrm{h}_{\mathrm{nn}}$ under certain conditions, are minimal for the system with end-product inhibition. In other words, of all possible patterns the simple case in Fig.la results in the least accumulation of the intermediates in the pathway. Thus, the same pattern that led to maximum overall gain minimizes the intermediate gains. As was noted earlier, this has important consequences in vivo where there is a limited solvent capacity to accommodate the increases in the concentrations of the metabolites (Atkinson, 1969). 
The two different classes of external effects that we have been considering are probably the most important and common perturbations with which such control systems must normally deal in the cell. However, there is one additional effect that should be considered: namely, the effect of variations in the exogenous supply of the end-product itself. When the end-product is available in the environment the control system should function to limit the flow of substrate into the pathway and thus spare the synthesis of the unnecessary intermediates. It can be demonstrated that the simple pattern of control by end-product inhibition is optimal also with respect to this criterion (manuscript in preparation). In contrast to the overall gain $\mathrm{L}_{\mathrm{nO}}$, to the intermediate gains $L_{i 0}$ and the sensitivity $S_{L_{n O}} h_{n n}$, the gains $\mathrm{L}_{\text {in' }}$ are a11 relatively independent of $\mathrm{g}_{1 \mathrm{n}}$ as long as $-\mathrm{g}_{1 \mathrm{n}} \geq \mathrm{h}_{\mathrm{nn}}$.

In summary, to satisfy the various and sometimes conflicting objectives for the design of a control system that are discussed in the preceding paragraphs, a strength of inhibition $-g_{1 n} \geq h_{n n}$ would appear to be required. With this choice of the parameter $g_{1 n}$, maximum sparing of the intermediates will be assured, and an optimal balance between maximum overall gain $\mathrm{L}_{\mathrm{nO}}$ and minimum sensitivity $\mathrm{S}_{\mathrm{L}_{\mathrm{n}}} \mathrm{h}_{\mathrm{nn}}$ can be achieved which slightly favors desensitization. Minimal accumulation of the metabolic intermediates is also favored by this choice of the parameter $g_{1 n}$.

Acknowledgments. This work was supported by a grant from the National Science Foundation (GB-27701).

\section{Appendix}

In arriving at the conclusion in Eq. (17) I used the fact that every term in the expansion of the determinant $(-1)^{n-1} \Delta_{n-1}$ is non-negative. In this appendix I shall prove this fact. By adding the bottom row of $\Delta_{n-1}$ from Eq. (9) to the row above it, then adding the resulting second row from the bottom to the third from the bottom, etc., the determinant $(-1)^{n-1} \Delta_{n-1}$ can be written as 


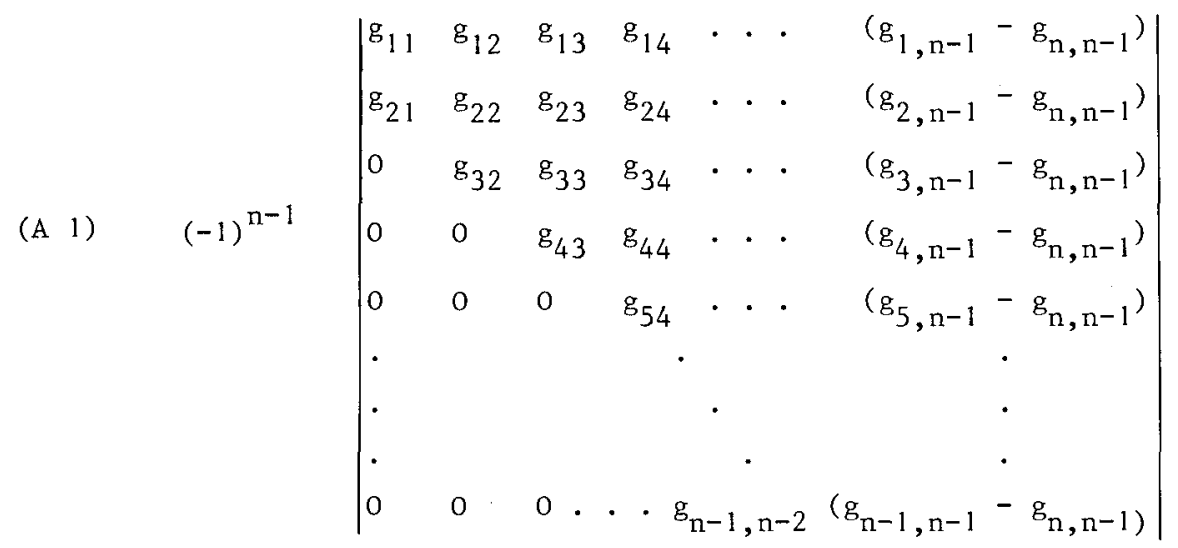

By expanding the cofactor of the first row and last column in Eq. (Al) the term producing the fraction of unity value in Eq. (17) is obtained. The fact that all terms in the expansion of this determinant are non-negative is seen by examining the form of the signs for the elements in the determinant.

A11 elements of the determinant on the main diagonal are negative, a11 those above are non-positive, the elements one space below the main diagonal are al1 positive, and all the remaining elements are zero. We must therefore prove that all the terms in the expansion of a determinant with the following signs are non-negative.

(A 2)

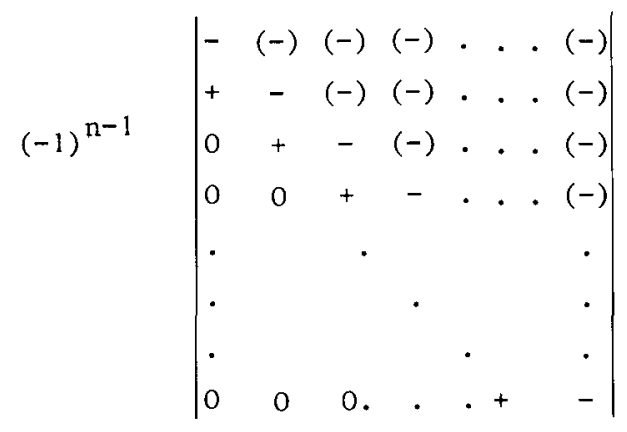

The proof is by induction. It is obviously true for the simple case with $\mathrm{n}=2$ :

$$
(-1)^{2-1}(-) \rightarrow(+)
$$

Assuming that it is true for the general case with $\mathrm{n}=\mathrm{m}-1$ we must show that it is true for $n=m$. If we expand the determinant represented in Eq. (A2) about the two elements in the last row we find

$$
\begin{aligned}
(-1)^{\mathrm{m}-1} \Delta_{\mathrm{m}-1}=-\left(\mathrm{g}_{\mathrm{m}-1, \mathrm{~m}-1}\right. & \left.-\mathrm{g}_{\mathrm{m}, \mathrm{m}-1}\right)(-1)^{\mathrm{m}-2} \Delta_{\mathrm{m}-2}^{\prime} \\
& +\mathrm{g}_{\mathrm{m}-1, \mathrm{~m}-2}(-1)^{\mathrm{m}-2} \Delta_{\mathrm{m}-2}^{\prime \prime}
\end{aligned}
$$


where the determinants $(-1)^{\mathrm{m}-2} \Delta_{\mathrm{m}-2}^{\prime}$ and $(-1)^{\mathrm{m}-2} \Delta_{\mathrm{m}-2}^{\prime \prime}$ are each of the form shown in Eq. (A2), except that the largest dimension is $\mathrm{m}-2$. Each of these determinants has only non-negative terms in its expansion by assumption. The element $g_{\mathrm{m}-1, \mathrm{~m}-2}>0$ and the element $\left(\mathrm{g}_{\mathrm{m}-1, \mathrm{~m}-1}-\mathrm{g}_{\mathrm{m}, \mathrm{m}-1}\right) \leq 0$; therefore the determinant $(-1)^{\mathrm{m}^{-1}} \Delta_{\mathrm{m}-1}$ must be expandable with only non-negative terms. This completes the proof by showing that it is true for $n=m$.

\section{$\underline{\text { References }}$}

Atkinson,D.E. (1969). Current topics in cellular regulation 1, 29 Koch,A.L. (1967). J.Theoret.Biol. 15, 75

Kohen, E.,Kohen, C. (1972). Federation Proc. 31, 467

Monod, J., Changeux, J .-P., Jacob, F. (1963). J.Mo1.Biol. 6, 306

Savageau, M.A. (1969). J.Theoret.Bio1. 25, 370

Savageau,M.A. (1971). Arch.Biochem.Biophys. 145, 612

Savageau,M.A. (1972). Current topics in cellular regulation 6, 63

Umbarger,H.E. (1956). Sci. 123, 848

Wong, J.T.-F., Hanes, C.S. (1962). Can.J.Biochem.Physio1. 40, 763

Yates,R.A.,Pardee,A.B. (1956). J.Biol.Chem. 221, 757

Dr. Michael A. Savageau

Department of Microbiology

The University of Michigan

Ann Arbor, Michigan 48104, USA 